\title{
Quadratic Filtering for Discrete-Time Systems with Measurement Delay and Packet Dropping
}

\author{
Ling Li, ${ }^{1}$ Lei Tan, ${ }^{1}$ Xinmin Song $\mathbb{D},{ }^{1}$ and Xuehua Yan $^{2}$ \\ ${ }^{1}$ The School of Information Science and Engineering, Shandong Normal University, Jinan 250014, China \\ ${ }^{2}$ School of Electrical Engineering, University of Jinan, Jinan, Shandong, China \\ Correspondence should be addressed to Xinmin Song; xinminsong@sina.com
}

Received 11 May 2020; Accepted 19 June 2020; Published 5 August 2020

Academic Editor: Jianquan Lu

Copyright $(0) 2020$ Ling Li et al. This is an open access article distributed under the Creative Commons Attribution License, which permits unrestricted use, distribution, and reproduction in any medium, provided the original work is properly cited.

We consider the problem of remote estimation with time delay and multiplicative noise for multichannel systems. First, we apply the reorganized innovation analysis approach to construct the original delay system into a new delay-free system. Secondly, the delay-free system will be reconstructed by the quadratic filtering method to obtain an augmented system. Then, Kalman filtering theory and projection formula are used to solve two Riccati equations and one Lyapunov equation for the augmented system, and the quadratic filter for the measurement delay system on the packet loss network can be obtained. Finally, we use a numerical example to illustrate the effectiveness of the method.

\section{Introduction}

In recent years, the problem of missing measurements caused by unreliable channel transmission has been the focus of many scholars [1-3]. The research on the problem of packet loss can be roughly divided into two directions: one is to solve the linear estimator based on the minimum mean square error method and the other is to use the quadratic filtering method. Nahi [4] believes that the observation sequence may contain only noise when the packets are lost and derives a set of recursive formulas similar to Kalman filtering in the sense of the minimum mean square error. In [5], the Kalman filter is implemented by intermittent observation to solve the problem of information loss in large wireless sensor networks. Zhang et al. [6] propose an estimator that can be applied to an infinite horizon, and its iteration only includes solving a Riccati equation. This estimator avoids the convergence analysis problem caused by the calculation of the Lyapunov equation in traditional estimation methods. The authors in [7] apply the recombination innovation analysis method to obtain an optimal linear filter, which solves the remote estimation problem of the packet loss network with the measurement delay system obeying Bernoulli distribution.
However, with the development of engineering technology [8-10], the performance of the traditional linear estimator cannot meet the requirements of the real system. Therefore, people are paying more and more attention to the optimization design and implementation of the estimator. De Santis et al. [11] first propose a method called quadratic filtering, which uses a quadratic function of the measurement equation to improve the performance of the filter. Experiments show that this method is superior to the linear filtering method in estimating performance. This research has attracted great attention of many scholars. CaballeroÁguila et al. [12] consider using innovative methods to solve the least square linear estimation problem and simplify the quadratic estimation problem into a linear estimation problem in a suitable augmented system. After that, Cacace et al. [13] add the packet loss factor to the measurement model and use the quadratic filtering method to obtain a filter iteration equation with a smaller estimation error. The Kronecker algebraic rules are used in [14] to discuss the stochastic properties of augmented noise in augmented systems. Then, the linear estimation of the discrete-time non-Gaussian system is obtained by the projection formula. Cacace et al. [15] propose a feedback quadratic filter that rewrites the system model by introducing an output 
injection term and prove that the performance of the feedback quadratic filter depends on the gain parameter of the output term.

Meanwhile, researchers found that time delay is common due to uncertain factors such as bandwidth and network failures [16-21]. Especially, the state estimation problem has received much attention for time-delay systems in [22-26]. The emergence of time delay often leads to the instability and even worsens the overall performance of the system. Therefore, solving the time-delay problem in life is bound to be an important subject of our research. The emergence of time delay often leads to the instability of the system and even makes the overall performance of the system worse, so solving the problem of time delay in life is bound to be the most important research content [27]. By solving a partial differential equation, the solution of a continuous observation delay system is obtained. The authors in [28] transform the discrete system with observation delay into a nonobservation delay system estimation problem by expanding the dimensions and then obtain the filter based on the standard Kalman filtering theory. Zhang et al. [29] propose a new-information reorganization analysis theory, that is, keeping observation information unchanged and then rearranging and combining observation data from different channels and different time delays into a system without time delay. Finally, the new observation data are introduced into the innovation sequence, and the signal is designed by using the projection theory. Song et al. [7] extended the abovementioned innovation restructuring theory to the study of infinite time estimation and made a deeper demonstration of the estimation of multistable systems.

Inspired by the above studies, this paper considers the quadratic filter problem of time-delay systems with multichannel multiplicative noise in discrete time. First of all, we assume that the measurement has a delay phenomenon, and the measurement is transmitted through multiple communication channels. The packet loss of each channel is described by the Bernoulli process of independent and identical distribution. Secondly, we use the innovation recombination theory to rearrange and combine the above observation data and obtain a new time-delay free observation system structure. Finally, we construct a quadratic filtering equation for the new time-delay free observation system and obtain a new filter by solving two Riccati equations and one Lyapunov equation. The main contribution of this paper is to effectively combine the quadratic filtering method with the innovation recombination theory; therefore, so as to obtain the quadratic filtering scheme of the discrete-time system with packet loss and measurement delay.

The rest of this article is organized as follows. First, Section 2 provides the question statement and preliminary. Section 3 provides quadratic filter solutions of the problem with detailed derivation processes. This part is the key result of this paper. Then, Section 4 is a simulation example to prove the effectiveness of the estimator algorithm in Section 3. Finally, the summary of this paper is given in Section 5 .
1.1. Notation. Throughout this technical paper, the superscripts " $T$ " and "-1" represent the transpose and inverse of a matrix, $\mathscr{R}^{n}$ denotes the $n$-dimensional Euclidean space, $\mathbb{E}\{\cdot\}$ stands for the mathematical expectation operator, $\otimes$ and $\odot$ are used to denote the Kronecker product and the Hadamard product, respectively, $I$ represents an identity matrix of the appropriate dimension, and $\delta_{i j}=0$ for $i \neq j$ and $\delta_{i i}=1$. We use $\operatorname{diag}\left\{\lambda_{1}, \ldots, \lambda_{n}\right\}$ to represent a diagonal matrix, where $\lambda_{1}, \ldots, \lambda_{n}$ are the diagonal elements of this diagonal matrix. If the dimensions are not explicitly stated, matrices are assumed to have compatible dimensions with algebraic operations.

\section{Problem Statement and Preliminary}

In this section, we consider a discrete time-delay system for which the state and measurement equations are as follows:

$$
\begin{aligned}
x(k+1) & =A(k) x(k)+n(k), \\
y_{0}(k) & =\xi(k) B_{0}(k) x(k)+v_{0}(k), \\
y_{1}(k) & =\theta(k) B_{1}(k) x(k-d)+v_{1}(k), \quad k \geq d,
\end{aligned}
$$

where $k=0,1,2,3, \ldots$ is the time instant, $d$ is the measurement delay time, $x(k) \in \mathscr{R}^{n}, \quad y_{0}(k) \in \mathscr{R}^{m_{1}}$, and $y_{1}(k) \in \mathscr{R}^{m_{2}}$ are, respectively, the system state and measurement, and $n(k), v_{0}(k)$, and $v_{1}(k)$ are the system noise and measurement noise with zero mean and covariances $\mathbb{E}\left\{n(k) n^{T}(j)\right\}=Q \delta_{k, j}, \quad \mathbb{E}\left\{v_{0}(k) v_{0}^{T}(j)\right\}=R_{0} \delta_{k, j}, \quad$ and $\mathbb{E}\left\{v_{1}(k) v_{1}^{T}(j)\right\}=R_{1} \delta_{k, j}, \quad$ respectively. Here, $\quad \xi(k)=$ $\operatorname{diag}\left\{\xi_{1}(k), \ldots, \xi_{m 1}(k)\right\} \quad$ and $\quad \theta(k)=\operatorname{diag}\left\{\theta_{1}(k), \ldots\right.$, $\left.\theta_{m 2}(k)\right\}$. The mutually uncorrelated and identically distributed (i.i.d.) Bernoulli random variables $\xi_{i}(k)$ and $\theta_{i}(k)$ are employed to describe, respectively. The packet loss phenomenon in the $m_{1}$ channels and $m_{2}$ channels is with $P_{r}\left\{\xi_{i}(k)=1\right\}=\alpha_{i}, P_{r}\left\{\xi_{i}(k)=0\right\}=1-\alpha_{i}, \quad P_{r}\left\{\theta_{i}(k)=1\right\}=$ $\beta_{i}$, and $P_{r}\left\{\theta_{i}(k)=0\right\}=1-\beta_{i}$. The initial state $x(0)$ is a random vector with mean $\mu_{0}$ and covariance matrix $\mathbb{E}\left\{\left[x(0)-\mu_{0}\right]\left[x(0)-\mu_{0}\right]^{T}\right\}=P_{0}$. It should be noted that for simplicity, we assume that $B_{0}(k)$ and $B_{1}(k)$ are constant matrices with appropriate dimensions. The random processes $n(k), v_{0}(k), v_{1}(k), \xi(k)$, and $\theta(k)$ are independent of the initial state $x(0)$.

For convenience, the measurement $y(k)$ can be rewritten as the following:

$$
y(k)= \begin{cases}y_{0}(k), & 0 \leq k<d, \\
{\left[\begin{array}{l}
y_{0}(k) \\
y_{1}(k)
\end{array}\right],} & k \geq d .\end{cases}
$$

2.1. Problem Statement. For the given systems (1) and (4), we try to construct the quadratic state and measurement vector and then get the quadratic filter iteration equation of the new system through the projection method and the basic theory of the Kalman filter. 


\section{Main Results}

Since there is a time delay $d$ at instant $k$, state $x(k-d)$ has an additional measurement $y_{1}(k)$. In addition, when $k \geq d$, the measurement $y(k)$ contains the time delay. According to [29], the linear space $\mathscr{L}\left\{\{y(s)\}_{s=0}^{k}\right\}$ contains the same information as $\mathscr{L}\left\{\left\{Y_{1}(s)\right\}_{s=0}^{k-d}\right\},\left\{\left\{Y_{0}(s)\right\}_{s=k-d+1}^{k}\right\}$, where the new observations $Y_{1}(s)$ and $Y_{0}(s)$ are provided as follows:

$$
\begin{aligned}
& Y_{1}(s)=\left[\begin{array}{c}
y_{0}(s) \\
y_{1}(s+d)
\end{array}\right], \quad 0 \leq s \leq k-d, \\
& Y_{0}(s)=y_{0}(s), \quad k-d<s \leq k .
\end{aligned}
$$

For convenience, $Y_{0}(s)$ and $Y_{1}(s)$ can be rewritten as

$$
\begin{aligned}
& Y_{1}(s)=H_{1} x(s)+V_{1}(s), \\
& Y_{0}(s)=H_{0} x(s)+V_{0}(s),
\end{aligned}
$$

where

$$
\begin{aligned}
H_{1} & =\left[\begin{array}{c}
\xi(s) B_{0} \\
\theta(s+d) B_{1}
\end{array}\right], \\
H_{0} & =\xi(s) B_{0}, \\
V_{1}(s) & =\left[\begin{array}{c}
v_{0}(s) \\
v_{1}(s+d)
\end{array}\right], \\
V_{0}(s) & =v_{0}(s) .
\end{aligned}
$$

Before introducing the quadratic filtering problem, we will construct the augmented state and measurement vectors by stacking the original vectors and obtaining their secondorder Kronecker powers. Then, we can get the new state vector and the measurement vector as shown below:

$$
x^{[2]}(s+1)=A^{[2]} x^{[2]}(s)+f(s)+m_{n(s)}^{(2)} \text {, }
$$

where

$$
f(s)=n^{[2]}(s)+A x(s) \otimes n(s)+n(s) \otimes A x(s)-m_{n(s)}^{(2)},
$$

with $E\{f(s)\}=0$.

Similarly, it is not difficult to obtain the following measurement equation:

$$
\begin{aligned}
& y_{0}^{[2]}(s)=\phi^{[2]} B_{0}^{[2]} x^{[2]}(s)+l(s)+m_{V_{0}(s)}^{[2]}, \\
& y_{1}^{[2]}(s)=\left[\begin{array}{ll}
\phi & 0 \\
0 & \varphi
\end{array}\right]^{[2]}\left[\begin{array}{l}
B_{0} \\
B_{1}
\end{array}\right]^{[2]} x^{[2]}(s)+g(s)+m_{V_{1}}^{(2)}(s)
\end{aligned}
$$

where

$$
\begin{aligned}
l(s) & =[\xi(s)-\phi]^{[2]} B_{0}^{[2]} x^{[2]}(s)+\left[\xi(s) B_{0} x(s)\right] \otimes V_{0}(s)+V_{0}(s) \otimes\left[\xi(s) B_{0} x(s)\right]+V_{0}^{[2]}(s)-m_{V_{0}(s)}^{(2)}, \\
g(s) & =\bar{H}_{1}^{[2]} x^{[2]}(s)+V_{1}^{[2]}(s)+H_{1} x(s) \otimes V_{1}(s)+V_{1}(s) \otimes H_{1} x(s)-m_{V_{1}(s)}^{(2)}, \\
\bar{H}_{1} & =\left[\begin{array}{cc}
\xi(s)-\phi & 0 \\
0 & \theta(s+d)-\varphi
\end{array}\right]\left[\begin{array}{c}
B_{0} \\
B_{1}
\end{array}\right],
\end{aligned}
$$

where

$$
\begin{aligned}
\phi^{[2]} & =\mathbb{E}\{\xi(s) \otimes \xi(s)\}=\left[\begin{array}{cccc}
\phi_{11} & 0 & \cdots & 0 \\
0 & \phi_{22} & \cdots & 0 \\
\vdots & \vdots & \ddots & \vdots \\
0 & 0 & \cdots & \phi_{m_{1} m_{1}}
\end{array}\right], \\
{\left[\begin{array}{ll}
\phi & 0 \\
0 & \varphi
\end{array}\right]^{[2]} } & =\mathbb{E}\left\{\left[\begin{array}{cc}
\xi(s) & 0 \\
0 & \theta(s)
\end{array}\right] \otimes\left[\begin{array}{ccc}
\xi(s) & 0 \\
0 & \theta(s)
\end{array}\right]\right\} \\
& =\left[\begin{array}{cccc}
\operatorname{diag}\left\{\phi_{11}, \varphi_{11}\right\} & 0 & \cdots & 0 \\
0 & \operatorname{diag}\left\{\phi_{22}, \varphi_{22}\right\} & \cdots & 0 \\
\vdots & \vdots & \ddots & \vdots \\
0 & 0 & \cdots & \varphi_{m_{2} m_{2}}
\end{array}\right],
\end{aligned}
$$

$$
\begin{aligned}
\phi_{11} & =\left[\begin{array}{cccc}
\alpha_{1} & 0 & \cdots & 0 \\
0 & \alpha_{1} \alpha_{2} & \cdots & 0 \\
\vdots & \vdots & \ddots & \vdots \\
0 & 0 & \cdots & \alpha_{1} \alpha_{m_{1}}
\end{array}\right], \\
\phi_{22} & =\left[\begin{array}{cccc}
\alpha_{2} \alpha_{1} & 0 & \cdots & 0 \\
0 & \alpha_{2} & \cdots & 0 \\
\vdots & \vdots & \ddots & \vdots \\
0 & 0 & \cdots & \alpha_{2} \alpha_{m_{1}}
\end{array}\right], \\
\phi_{m_{1} m_{1}} & =\left[\begin{array}{cccc}
\alpha_{m_{1}} \alpha_{1} & 0 & \cdots & 0 \\
0 & \alpha_{m_{1}} \alpha_{2} & \cdots & 0 \\
\vdots & \vdots & \ddots & \vdots \\
0 & 0 & \cdots & \alpha_{m_{1}}
\end{array}\right],
\end{aligned}
$$

in which 


$$
\begin{aligned}
& \varphi_{11}=\left[\begin{array}{cccc}
\alpha_{1} \beta_{1} & 0 & \cdots & 0 \\
0 & \alpha_{1} \beta_{1} & \cdots & 0 \\
\vdots & \vdots & \ddots & \vdots \\
0 & 0 & \cdots & \alpha_{1} \beta_{m_{2}}
\end{array}\right], \\
& \varphi_{22}=\left[\begin{array}{cccc}
\alpha_{2} \beta_{1} & 0 & \cdots & 0 \\
0 & \alpha_{2} \beta_{2} & \cdots & 0 \\
\vdots & \vdots & \ddots & \vdots \\
0 & 0 & \cdots & \alpha_{2} \beta_{m_{2}}
\end{array}\right] \text {, } \\
& \varphi_{m_{2} m_{1}}=\left[\begin{array}{cccc}
\beta_{1} \alpha_{1} & 0 & \cdots & 0 \\
0 & \beta_{1} \alpha_{2} & \cdots & 0 \\
\vdots & \vdots & \ddots & \vdots \\
0 & 0 & \cdots & \beta_{1} \beta_{m_{2}}
\end{array}\right] \text {, } \\
& \varphi_{m_{2} m_{2}}=\left[\begin{array}{cccc}
\beta_{m_{2}} \alpha_{1} & 0 & \cdots & 0 \\
0 & \beta_{m_{2}} \alpha_{2} & \cdots & 0 \\
\vdots & \vdots & \ddots & \vdots \\
0 & 0 & \cdots & \beta_{m_{2}}
\end{array}\right] \text {, }
\end{aligned}
$$

with $\phi=\operatorname{diag}\left\{\alpha_{1}, \ldots, \alpha_{m_{1}}\right\}, \varphi=\operatorname{diag}\left\{\beta_{1}, \ldots, \beta_{m_{2}}\right\}, E\{l(s)\}=$ 0 , and $E\{g(s)\}=0$.

Then, the new state vector and the measurement vector can be written as

$$
\begin{aligned}
& \mathbf{x}(s) \triangleq\left[\begin{array}{c}
x(s) \\
x^{[2]}(s)
\end{array}\right], \\
& \mathbf{y}(s) \triangleq\left[\begin{array}{c}
y(s) \\
y^{[2]}(s)
\end{array}\right] .
\end{aligned}
$$

Finally, we can derive the augmented system as follows:

$$
\begin{aligned}
\mathbf{x}(s+1) & =\mathbf{A} \mathbf{x}(s)+\mathbf{C U}_{0}(s)+\mathbf{F}(s), \\
\mathbf{Y}_{0}(s) & =\mathbf{H}_{0} \mathbf{x}(s)+\mathbf{E}_{0} \mathbf{U}_{0}(s)+\mathbf{L}(s), \\
\mathbf{Y}_{1}(s) & =\mathbf{H}_{1} \mathbf{x}(s)+\mathbf{E}_{1} \mathbf{U}_{1}(s)+\mathbf{G}(s),
\end{aligned}
$$

where

$$
\begin{aligned}
\mathbf{A} & \triangleq\left[\begin{array}{cc}
A & 0 \\
0 & A^{[2]}
\end{array}\right], \\
\mathbf{C} & \triangleq\left[\begin{array}{cc}
0 & 0 \\
I_{n^{2} \times n^{2}} & 0
\end{array}\right], \\
\mathbf{F}(s) & \triangleq\left[\begin{array}{c}
n(s) \\
f(s)
\end{array}\right], \\
\mathbf{H}_{0} & \triangleq\left[\begin{array}{cc}
\phi B_{0} & 0 \\
0 & \phi^{[2]} B_{0}^{[2]}
\end{array}\right], \\
\mathbf{E}_{0} & \triangleq\left[\begin{array}{cc}
0 & 0 \\
0 & I_{m_{1}^{2} \times m_{1}^{2}}
\end{array}\right],
\end{aligned}
$$

$$
\begin{aligned}
& \mathbf{U}_{0}(s) \triangleq\left[\begin{array}{c}
m_{n(s)}^{(2)} \\
m_{V_{0}}^{(2)}(s)
\end{array}\right], \\
& \mathbf{L}(s) \triangleq\left[\begin{array}{c}
(\xi(s)-\phi) B_{0} x(s)+V_{0}(s) \\
l(s)
\end{array}\right], \\
& \mathbf{U}_{1}(s) \triangleq\left[\begin{array}{c}
m_{n(s)}^{(2)} \\
m_{V_{1}}^{(2)}(s)
\end{array}\right] \text {, } \\
& \mathbf{E}_{1} \triangleq\left[\begin{array}{cc}
0 & 0 \\
0 & I_{m_{2}^{2} \times m_{2}^{2}}
\end{array}\right] \text {, } \\
& \mathbf{G}(s) \triangleq\left[\begin{array}{c}
\bar{H}_{1} x(s)+V_{1}(s) \\
g(s)
\end{array}\right], \\
& \mathbf{H}_{1} \triangleq\left[\begin{array}{c}
{\left[\begin{array}{ll}
\phi & 0 \\
0 & \varphi
\end{array}\right]\left[\begin{array}{l}
B_{0} \\
B_{1}
\end{array}\right]} \\
0 \\
0
\end{array}\left[\begin{array}{ll}
\phi & 0 \\
0 & \varphi
\end{array}\right]^{[2]}\left[\begin{array}{l}
B_{0} \\
B_{1}
\end{array}\right]^{[2]}\right] .
\end{aligned}
$$

Note that the new measurements $\mathbf{Y}_{0}(s)$ and $\mathbf{Y}_{1}(s)$ are delay free. $\{\mathbf{F}(s)\},\{\mathbf{L}(s)\}$, and $\{\mathbf{G}(s)\}$ for all $s$ and the initial $\mathbf{x}(0)$ are mutually independent. Moreover, $\{\mathbf{F}(s)\},\{\mathbf{L}(s)\}$, and $\{\mathbf{G}(s)\}$ are zero mean such that $\mathbb{E}\left\{\mathbf{F}(s) \mathbf{F}^{T}(j)\right\}=$ $\mathbf{Q}_{F(s)} \delta_{s, j}, \mathbb{E}\left\{\mathbf{L}(s) \mathbf{L}^{T}(j)\right\}=\mathbf{Q}_{L(s)} \delta_{s, j}$, and $\mathbb{E}\left\{\mathbf{G}(s) \mathbf{G}^{T}(j)\right\}=$ $\mathbf{Q}_{G(s)} \delta_{s, j}$, and the detailed calculation processes are given below:

$$
\begin{aligned}
\mathbf{Q}_{F(s)} & =\left[\begin{array}{cc}
\mathbb{E}\left\{n(s) n^{T}(s)\right\} & \mathbb{E}\left\{n(s) f^{T}(s)\right\} \\
\mathbb{E}\left\{f(s) n^{T}(s)\right\} & \mathbb{E}\left\{f(s) f^{T}(s)\right\}
\end{array}\right] \\
& =\left[\begin{array}{cc}
\mathbb{E}\left\{n(s) n^{T}(s)\right\} & \mathbb{E}\left\{n(s) n^{[2] T}(s)\right\} \\
\mathbb{E}\left\{n^{[2]}(s) n^{T}(s)\right\} & *_{1}
\end{array}\right],
\end{aligned}
$$

where

$$
\begin{aligned}
*_{1}= & \mathbb{E}\left\{\left[n(s) n^{T}(s)\right] \otimes\left[n(s) n^{T}(s)\right]\right\}-m_{n(s)}^{(2)} m_{n(s)}^{(2) T} \\
& +(I+\Pi)\left[\left(A D(s)^{T}\right) \otimes \mathbb{E}\left\{n(s) n^{T}(s)\right\}\right](I+\Pi) .
\end{aligned}
$$

It should be pointed out that the entries of $\mathbb{E}\left\{n(s) n^{[2] T}(s)\right\}, \quad \mathbb{E}\left\{\left[n(s) n^{T}(s)\right] \otimes\left[n(s) n^{T}(s)\right]\right\}, \quad$ and $\mathbb{E}\left\{n(s) n^{T}(s)\right\}$ are known since they are the elements of $m_{n(s)}^{(3)}$, $m_{n(s)}^{(4)}$, and $m_{n(s)}^{(2)}$, respectively.

For convenience, let us define

$$
\begin{aligned}
& D^{(0,2)}(s) \triangleq \mathbb{E}\left\{x^{[2]}(s)\right\}, \\
& D^{(1,2)}(s) \triangleq \mathbb{E}\left\{x(s) x^{[2] T}(s)\right\}, \\
& D^{(2,2)}(s) \triangleq \mathbb{E}\left\{x^{[2]}(s) x^{[2] T}(s)\right\} .
\end{aligned}
$$

Then, we can calculate that 


$$
\begin{aligned}
D^{(0,2)}(s+1) & =\mathbb{E}\left\{x^{[2]}(s+1)\right\}=\operatorname{vec}^{T}(D(s+1)), \\
D^{(1,2)}(s+1) & =\mathbb{E}\left\{x(s+1) x^{[2] T}(s+1)\right\} \\
& =\mathbb{E}\left\{[A x(s)+n(s)]\left[A^{[2]} x^{[2]}(s)+f(s)+m_{n(s)}^{(2)}\right]\right\} \\
& =A D^{(1,2)}(s) A^{[2] T}+\mathbb{E}\left\{n(s) n^{[2] T}(s)\right\}, \\
D^{(2,2)}(s+1) & =\mathbb{E}\left\{x^{[2]}(s+1) x^{[2] T}(s+1)\right\} \\
& =\mathbb{E}\left\{\left[A^{[2]} x^{[2]}(s)+f(s)+m_{n(s)}^{(2)}\right] \times\left[A^{[2]} x^{[2]}(s)+f(s)+m_{n(s)}^{(2)}\right]\right\} \\
& =A^{[2]} D^{(2,2)}(s) A^{[2] T}+\mathbb{E}\left\{n^{[2]}(s) n^{[2] T}(s)\right\}+(I+\Pi) \times\left[\left(A D(s) A^{T}\right) \otimes \mathbb{E}\left\{n(s) n^{T}(s)\right\}\right](I+\Pi),
\end{aligned}
$$

where $\Pi$ is the matrix which guarantees that where $n(s) \otimes \operatorname{Ax}(s)=\Pi(\operatorname{Ax}(s) \otimes n(s))$.

Following the similar way for $\mathbf{Q}_{F(s)}$, one has

$$
\begin{aligned}
\mathbf{Q}_{L(s)} & =\mathbb{E}\left\{\mathbf{L}(s) \mathbf{L}^{T}(s)\right\} \\
& =\left[\begin{array}{cc}
\Gamma_{1} \odot\left(B_{0} D(s) B_{0}^{T}\right)+\mathbb{E}\left\{V_{0}(s) V_{0}^{T}(s)\right\} & *_{2} \\
*{ }_{2}^{T} & *_{3}
\end{array}\right], \\
\mathbf{Q}_{G(s)} & =\mathbb{E}\left\{\mathbf{G}(s) \mathbf{G}^{T}(s)\right\} \\
& =\left[\begin{array}{cc}
\operatorname{diag}\left\{\Gamma_{1}, \Lambda_{1}\right\} \odot\left(B_{1} D(s) B_{1}^{T}\right)+\mathbb{E}\left\{V_{1}(s) V_{1}^{T}(s)\right\} & *_{4} \\
*_{4}^{T} & *_{5}
\end{array}\right],
\end{aligned}
$$

$$
\begin{aligned}
*_{2}= & \mathbb{E}\left\{V_{0}(s) V_{0}^{[2] T}(s)\right\}+\mathbb{E}\left\{[\xi(s)-\phi][\xi(s)-\phi]^{[2] T}\right\} B_{0} D^{(1,2)}(s) B_{0}^{[2] T}, \\
*_{3}= & \left(\Gamma_{1} \otimes \Gamma_{1}\right) \odot\left(B_{0}^{[2]} D^{(2,2)}(s) B_{0}^{[2] T}\right)+\mathbb{E}\left\{V_{0}^{[2]}(s) V_{0}^{[2] T}(s)\right\}-m_{V_{0}(s)}^{(2)} m_{V_{0}}^{(2) T}(s) \\
& +\mathbb{E}\left\{(\xi(s)-\phi)^{[2]}\right\} B_{0}^{[2]} D^{(0,2)}(s) m_{V_{0}(s)}^{(2) T}+m_{V_{0}(s)}^{(2)} D^{(0,2) T}(s) B_{0}^{[2] T} \mathbb{E}\left\{(\xi(s)-\phi)^{[2] T}\right\} \\
& +\left(I+\Pi_{1}\right)\left[\Gamma_{1} \odot\left(B_{0} D(s) B_{0}^{T}\right) \otimes \mathbb{E}\left\{V_{0}(s) V_{0}^{T}(s)\right\}\right]\left(I+\Pi_{1}\right), \\
*_{4}= & \mathbb{E}\left\{\bar{H}_{2} \bar{H}_{2}^{[2] T}\right\}\left(B D^{(1,2)}(s) B^{[2] T}\right)+\mathbb{E}\left\{V_{1}(s) V_{1}^{[2] T}(s)\right\}, \\
*_{5}= & \left(\operatorname{diag}\left\{\Gamma_{1}, \Lambda_{1}\right\} \otimes \operatorname{diag}\left\{\Gamma_{1}, \Lambda_{1}\right\}\right) \odot\left(B_{1}^{[2]} D^{(2,2)}(s) B_{1}^{[2] T}\right)+\mathbb{E}\left\{V_{1}^{[2]}(s) V_{1}^{[2] T}(s)\right\} \\
& +\mathbb{E}\left\{\bar{H}_{2}^{[2]}\right\} B^{[2]} D^{(0,2)}(s) m_{V_{1}}^{(2) T}(s)+m_{V_{1}(s)}^{(2)} D^{(0,2) T}(s) B^{[2] T} \mathbb{E}\left\{\bar{H}_{2}^{[2] T}\right\}-m_{V_{1}(s)}^{(2)} m_{V_{1}(s)}^{(2) T} \\
& \left.+\left(I+\Pi_{2}\right)\left[\left(\operatorname{diag}\left\{\Gamma_{1}, \Lambda_{1}\right\}\right)\right) \odot\left(B_{1}^{[2]} D^{(2,2)}(s) B_{1}^{[2] T}\right) \otimes \mathbb{E}\left\{V_{1}(s) V_{1}^{T}(s)\right\}\right]\left(I+\Pi_{2}\right),
\end{aligned}
$$

where

$$
\begin{aligned}
& \Gamma_{1}=\left[\begin{array}{cccc}
\alpha_{1}\left(1-\alpha_{1}\right) & 0 & \cdots & 0 \\
0 & \alpha_{2}\left(1-\alpha_{2}\right) & \cdots & 0 \\
\vdots & \vdots & \ddots & \vdots \\
0 & 0 & \cdots & \alpha_{m_{1}}\left(1-\alpha_{m_{1}}\right)
\end{array}\right], \\
& \Lambda_{1}=\left[\begin{array}{cccc}
\beta_{1}\left(1-\beta_{1}\right) & 0 & \cdots & 0 \\
0 & \beta_{2}\left(1-\beta_{2}\right) & \cdots & 0 \\
\vdots & \vdots & \ddots & \vdots \\
0 & 0 & \cdots & \beta_{m_{2}}\left(1-\beta_{m_{2}}\right)
\end{array}\right] \text {, } \\
& \bar{H}_{2}=\left[\begin{array}{cc}
\xi(s)-\phi & 0 \\
0 & \theta(s+d)-\varphi
\end{array}\right] \text {, } \\
& B=\left[\begin{array}{l}
B_{0} \\
B_{1}
\end{array}\right] \text {. }
\end{aligned}
$$

It should be noted that $\Pi_{1}$ and $\Pi_{2}$ are the matrices which ensure $V_{0}(s) \otimes H_{0} x(s)=\Pi_{1}\left(H_{0} x(s) \otimes V_{0}(s)\right)$ and $V_{1}(s) \otimes$ $H_{1} x(s)=\Pi_{2}\left(H_{1} x(s) \otimes V_{1}(s)\right)$. Then, notice that the entries of $\mathbb{E}\left\{V_{0}(s) V_{0}^{T}(s)\right\}, \mathbb{E}\left\{\left[V_{0}(s) V_{0}^{T}(s)\right] \otimes\left[V_{0}(s) V_{0}^{T}(s)\right]\right\}, \mathbb{E}\left\{V_{0}^{[2]}\right.$ $\left.(s) V_{0}^{T}(s)\right\}, \mathbb{E}\left\{V_{1}(s) V_{1}^{T}(s)\right\}, \mathbb{E}\left\{V_{1}^{[2]}(s) V_{1}^{T}(s)\right\}$, and $\mathbb{E}\left\{\left[V_{1}\right.\right.$ $\left.\left.(s) V_{1}^{T}(s)\right] \otimes\left[V_{1}(s) V_{1}^{T}(s)\right]\right\}$ are known because they are the elements of $m_{V_{0}(s)}^{(2)}, m_{V_{0}(s)}^{(4)}, m_{V_{0}(s)}^{(3)}, m_{V_{1}(s)}^{(2)}, m_{V_{1}(s)}^{(3)}$, and $m_{V_{1}(s)}^{(4)}$, respectively.

We define the quadratic state estimator $\widehat{\mathbf{x}}(s+1 \mid s)$ as the projection of $\mathbf{x}(s+1)$ onto the linear space $\mathscr{L}\left\{\left\{\mathbf{Y}_{1}(i)\right\}_{i=0}^{k-d},\left\{\mathbf{Y}_{0}(i)\right\}_{i=k-d+1}^{k}\right\}$. In order to derive the projection, we give the following definitions of the innovation sequence: 


$$
\begin{aligned}
& \mathbf{w}(s, 0)=\mathbf{Y}_{0}(s)-\widehat{\mathbf{Y}}_{0}(s)=\mathbf{H}_{0} \widetilde{\mathbf{x}}(s)+\mathbf{L}(s), \\
& \mathbf{w}(s, 1)=\mathbf{Y}_{1}(s)-\widehat{\mathbf{Y}}_{1}(s)=\mathbf{H}_{1} \widetilde{\mathbf{x}}(s)+\mathbf{G}(s),
\end{aligned}
$$

where $\widehat{\mathbf{Y}}_{1}(s, 1)$ is the projection of $Y_{1}(s)$ onto the linear space of $\mathscr{L}\left\{\left\{\mathbf{Y}_{1}(i)\right\}_{i=0}^{s-1}\right\}$ and $\widehat{\mathbf{Y}}_{0}(s, 0)$ is the projection of $\mathbf{Y}_{0}(s)$ onto the linear space of $\mathscr{L}\left\{\left\{\mathbf{Y}_{1}(i)\right\}_{i=0}^{k-d},\left\{\mathbf{Y}_{0}(i)\right\}_{i=k-d+1}^{k}\right\}$. Then, we define $\widetilde{\mathbf{x}}(s, 0)=\mathbf{x}(s)-\widehat{\mathbf{x}}(s, 0)$ and $\widetilde{\mathbf{x}}(s, 1)=$ $\mathbf{x}(s)-\widehat{\mathbf{x}}(s, 1)$. It should be noted that the definitions of $\widehat{\mathbf{x}}(s, 0)$ and $\widehat{\mathbf{x}}(s, 1)$ are similar to $\widehat{\mathbf{Y}}_{0}(s, 0)$ and $\widehat{\mathbf{Y}}_{1}(s, 1)$. In addition, we reckon that $\left\{\{\mathbf{w}(i, 1)\}_{i=0}^{k-d},\{\mathbf{w}(i, 0)\}_{i=k-d+1}^{k}\right\}$ is an independent white noise and spans the same linear space as $\mathscr{L}\left\{\left\{\mathbf{Y}_{1}(i)\right\}_{i=0}^{k-d},\left\{\mathbf{Y}_{0}(i)\right\}_{i=k-d+1}^{k}\right\}$. Next, we derive the covariance $\mathbf{R}_{w(s, 0)}$ and $\mathbf{R}_{w(s, 1)}$ of the innovation sequence. For convenience, the following definitions are given:

$$
\begin{aligned}
& \mathbf{P}_{0}(s) \triangleq \mathbb{E}\left\{\widetilde{\mathbf{x}}(s, 0) \widetilde{\mathbf{x}}^{T}(s, 0)\right\}, \\
& \mathbf{P}_{1}(s) \triangleq \mathbb{E}\left\{\widetilde{\mathbf{x}}(s, 1) \widetilde{\mathbf{x}}^{T}(s, 1)\right\}, \\
& \mathbf{D}(s) \triangleq \mathbb{E}\left\{\mathbf{x}(s) \mathbf{x}^{T}(s)\right\} .
\end{aligned}
$$

Then, the covariance matrices of the innovation sequences (26) and (27) can be derived by the following formula:

$$
\begin{aligned}
& \mathbf{R}_{w(s, 0)}=\mathbb{E}\left\{\mathbf{w}(s, 0) \mathbf{w}^{T}(s, 0)\right\}=\mathbf{H}_{0} \mathbf{P}_{0}(s) \mathbf{H}_{0}^{T}+\mathbf{Q}_{L(s)}, \\
& \mathbf{R}_{w(s, 1)}=\mathbb{E}\left\{\mathbf{w}(s, 1) \mathbf{w}^{T}(s, 1)\right\}=\mathbf{H}_{1} \mathbf{P}_{1}(s) \mathbf{H}_{1}^{T}+\mathbf{Q}_{G(s)} .
\end{aligned}
$$

Finally, the covariance matrices $\mathbf{P}_{0}(s+1)$ and $\mathbf{P}_{1}(s+1)$ can be derived as

$$
\mathbf{P}_{1}(s+1)=\mathbf{A P}_{1}(s) \mathbf{A}^{T}+\mathbf{Q}_{F(s)}-\mathbf{A} \mathbf{P}_{1}(s) \mathbf{H}_{1}^{T} \mathbf{R}_{w(s, 1)}^{-1} \mathbf{H}_{1} \mathbf{P}_{1}(s) \mathbf{A}^{T},
$$

$$
\begin{aligned}
\mathbf{P}_{1}(0) & =\mathbf{D}_{1}(0), \\
\mathbf{P}_{0}(s+1) & =\mathbf{A P}_{0}(s) \mathbf{A}^{T}+\mathbf{Q}_{F(s)}-\mathbf{A} \mathbf{P}_{0}(s) \mathbf{H}_{0}^{T} \mathbf{R}_{w(s, 0)}^{-1} \mathbf{H}_{0} \mathbf{P}_{0}(s) \mathbf{A}^{T},
\end{aligned}
$$

$$
\mathbf{P}_{0}(k-d+1)=\mathbf{P}_{1}(k-d+1),
$$

and the Lyapunov equation $\mathbf{D}(s+1)$ can be calculated by

$$
\mathbf{D}(s+1)=\mathbf{A D}(s) \mathbf{A}^{T}+\mathbf{Q}_{F(s)} \text {. }
$$

Theorem 1. For given systems (15)-(17), the quadratic filter $\widehat{\mathbf{x}}(k \mid k)$ can be derived as

$$
\begin{aligned}
\widehat{\mathbf{x}}(k \mid k)= & \widehat{\mathbf{x}}(k, 0)+\mathbf{P}_{0}(k) \mathbf{H}_{0}^{T} \mathbf{R}_{w(k, 0)}^{-1}\left[\mathbf{Y}_{0}(k)-\mathbf{H}_{0} \widehat{\mathbf{x}}(k)\right. \\
& \left.-\mathbf{E}_{0} \mathbf{U}_{0}(k)\right]
\end{aligned}
$$

where the estimator $\widehat{\mathbf{x}}(k \mid 0)$ is computed by

$$
\begin{aligned}
\widehat{\mathbf{x}}(s+1,0)= & \mathbf{A} \widehat{\mathbf{x}}(s, 0)+\mathbf{C U}_{0}(s)+\mathbf{A P}_{0}(s) \mathbf{H}_{0}^{T} \mathbf{R}_{w(s, 0)}^{-1}\left[\mathbf{Y}_{0}(s)\right. \\
& \left.-\mathbf{H}_{0} \widehat{\mathbf{x}}(s)-\mathbf{E}_{0} \mathbf{U}_{0}(s)\right],
\end{aligned}
$$

with the initial value $\widehat{\mathbf{x}}(k-d+1,0)=\widehat{\mathbf{x}}(k-d+1,1)$, and $\widehat{\mathbf{x}}(k-d+1,1)$ is obtained by

$$
\begin{aligned}
\widehat{\mathbf{x}}(s+1,1)= & \mathbf{A} \widehat{\mathbf{x}}(s, 1)+\mathbf{C U}_{0}(s)+\mathbf{A} \mathbf{P}_{1}(s) \mathbf{H}_{1}^{T} \mathbf{R}_{w(s, 1)}^{-1}\left[\mathbf{Y}_{1}(s)\right. \\
& \left.-\mathbf{H}_{1} \widehat{\mathbf{x}}(s)-\mathbf{E}_{1} \mathbf{U}_{1}(s)\right] .
\end{aligned}
$$

Proof. According to (15), we can directly prove (38) by the projection theorem:

$$
\begin{aligned}
\widehat{\mathbf{x}}(s+1,0)= & \mathbf{A} \widehat{\mathbf{x}}(s, 0)+\mathbb{E}\left\{\mathbf{x}(s+1) \mathbf{w}^{T}(s, 0)\right\} \mathbf{R}_{w(s, 0)}^{-1} \mathbf{w}(s, 0) \\
= & \mathbf{A} \widehat{\mathbf{x}}(s, 0)+\mathbf{C U}_{0}(s)+\mathbf{A} \mathbf{P}_{0}(s) \mathbf{H}_{0}^{T} \mathbf{R}_{w(s, 0)}^{-1} \\
& \times\left[\mathbf{Y}_{0}(s)-\mathbf{H}_{0} \widehat{\mathbf{x}}(s)-\mathbf{E}_{0} \mathbf{U}_{0}(s)\right] .
\end{aligned}
$$

Then, we obtain the estimate error $\widetilde{\mathbf{x}}(s+1,0)$ by subtracting (38) from (15):

$$
\widetilde{\mathbf{x}}(s+1,0)=\mathbf{A} \widetilde{\mathbf{x}}(s, 0)+\mathbf{F}(s)-\mathbf{A P}_{0}(s) \mathbf{H}_{0}^{T} \mathbf{R}_{w(s, 0)}^{-1} \mathbf{w}(s, 0) .
$$

Therefore, the prediction error covariance $\mathbf{P}_{0}(s+1)$ can be calculated from (39):

$$
\begin{aligned}
\mathbf{P}_{0}(s+1) & =\mathbb{E}\left\{\widetilde{\mathbf{x}}(s+1,0) \widetilde{\mathbf{x}}^{T}(s+1,0)\right\} \\
& =\mathbf{A P}_{0}(s) \mathbf{A}^{T}+\mathbf{Q}_{F(s)}-\mathbf{A} \mathbf{P}_{0}(s) \mathbf{H}_{0}^{T} \mathbf{R}_{w(s, 0)}^{-1} \mathbf{H}_{0} \mathbf{P}_{0}(s) \mathbf{A}^{T} .
\end{aligned}
$$

Similar to formula (39), we can deduce (41) as follows:

$$
\begin{aligned}
\widehat{\mathbf{x}}(s+1,1)= & \mathbf{A} \widehat{\mathbf{x}}(s, 1)+\mathbb{E}\left\{\mathbf{x}(s+1) \mathbf{w}^{T}(s, 1)\right\} \mathbf{R}_{w(s, 0)}^{-1} \mathbf{w}(s, 1) \\
= & \mathbf{A} \widehat{\mathbf{x}}(s, 1)+\mathbf{C} \mathbf{U}_{0}(s)+\mathbf{A} \mathbf{P}_{1}(s) \mathbf{H}_{0}^{T} \mathbf{R}_{w(s, 1)}^{-1} \\
& \times\left[\mathbf{Y}_{1}(s)-\mathbf{H}_{1} \widehat{\mathbf{x}}(s)-\mathbf{E}_{1} \mathbf{U}_{1}(s)\right] .
\end{aligned}
$$

By combining (15) and (41), one has

$$
\widetilde{\mathbf{x}}(s+1,1)=\mathbf{A} \widetilde{\mathbf{x}}(s, 1)+\mathbf{F}(s)-\mathbf{A P}_{1}(s) \mathbf{H}_{1}^{T} \mathbf{R}_{w(s, 1)}^{-1} \mathbf{w}(s, 1) .
$$

As such, we get the Riccati equation:

$$
\begin{aligned}
\mathbf{P}_{1}(s+1)= & \mathbb{E}\left\{\widetilde{\mathbf{x}}(s+1,1) \widetilde{\mathbf{x}}^{T}(s+1,1)\right\} \\
= & \mathbf{A P}_{1}(s) \mathbf{A}^{T}+\mathbf{Q}_{F(s)}-\mathbf{A P}_{1}(s) \mathbf{H}_{1}^{T} \mathbf{R}_{w(s, 1)}^{-1} \\
& \times \mathbf{H}_{1} \mathbf{P}_{1}(s) \mathbf{A}^{T} .
\end{aligned}
$$

By the definition of $\widehat{\mathbf{x}}(k-d+1,0)$ and $\widehat{\mathbf{x}}(k-d+1,1)$, we can conclude that $\widehat{\mathbf{x}}(k-d+1,0)=\widehat{\mathbf{x}}(k-d+1,1)$. Therefore, formula (33) is proved.

The proof is finished. 


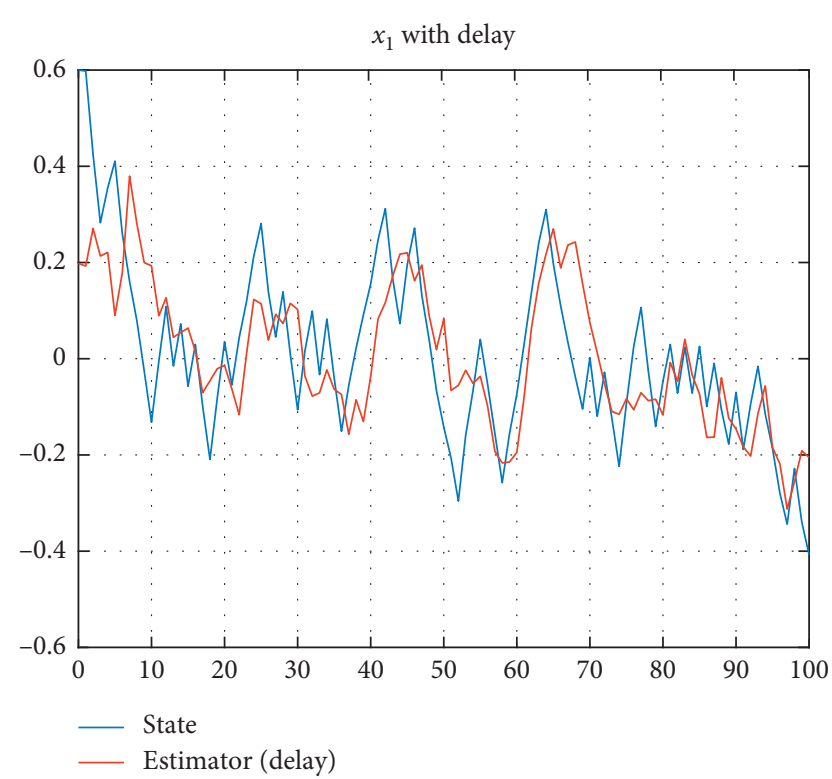

Figure 1: The first state component $x_{1}(k)$ and the filter $\hat{x}(k \mid k)$ (delay).

\section{Simulation Results}

In this section, we demonstrate the effectiveness of the proposed algorithm by a simulation example. The linear discrete-time system can be written as follows:

$$
\begin{aligned}
x(k+1) & =\left[\begin{array}{cc}
0.88 & 0.1 \\
0.45 & 0.28
\end{array}\right] x(k)+n(k), \\
y_{0}(k) & =\xi(k)\left[\begin{array}{ll}
1 & 2 \\
2 & 1
\end{array}\right] x(k)+v_{0}(k), \\
y_{1}(k) & =\theta(k)\left[\begin{array}{ll}
2 & 1 \\
1 & 2
\end{array}\right] x(k)+v_{1}(k),
\end{aligned}
$$

with initial values given as $x(0)=\left[\begin{array}{c}0.6 \\ -0.3\end{array}\right], \widehat{x}(0)=$ $\left[\begin{array}{l}0 \\ 0\end{array}\right], Q=\left[\begin{array}{cc}0.01 & 0 \\ 0 & 0.0225\end{array}\right], D(0)=P(0)=\left[\begin{array}{cc}1.08 & 0 \\ 0 & 0.03\end{array}\right]$, $\phi=\operatorname{diag}\{0.6,0.7\}, \varphi=\operatorname{diag}\{0.7,0.9\}, R_{0}=\left[\begin{array}{cc}0.0625 & 0 \\ 0 & 0.16\end{array}\right]$, and $R_{1}=\left[\begin{array}{cc}0.04 & 0 \\ 0 & 0.09\end{array}\right] ; n(k), v_{0}(k)$, and $v_{1}(k)$ are the

white noises with zero mean and covariances $Q, R_{0}$, and $R_{1}$, respectively.

We use MATLAB to simulate the performance of the estimator in Theorem 1, and the numerical results are shown in Figures 1-4. First, it can be seen that Figures 1 and 2 represent quadratic estimators with measurement

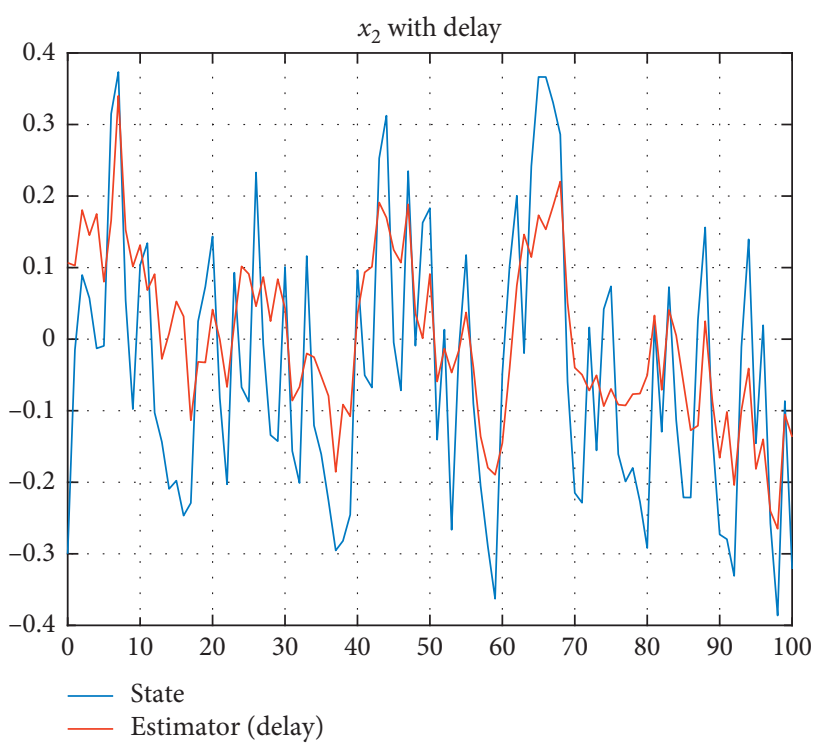

FIgURE 2: The second state component $x_{2}(k)$ and the filter $\widehat{x}(k \mid k)$ (delay).

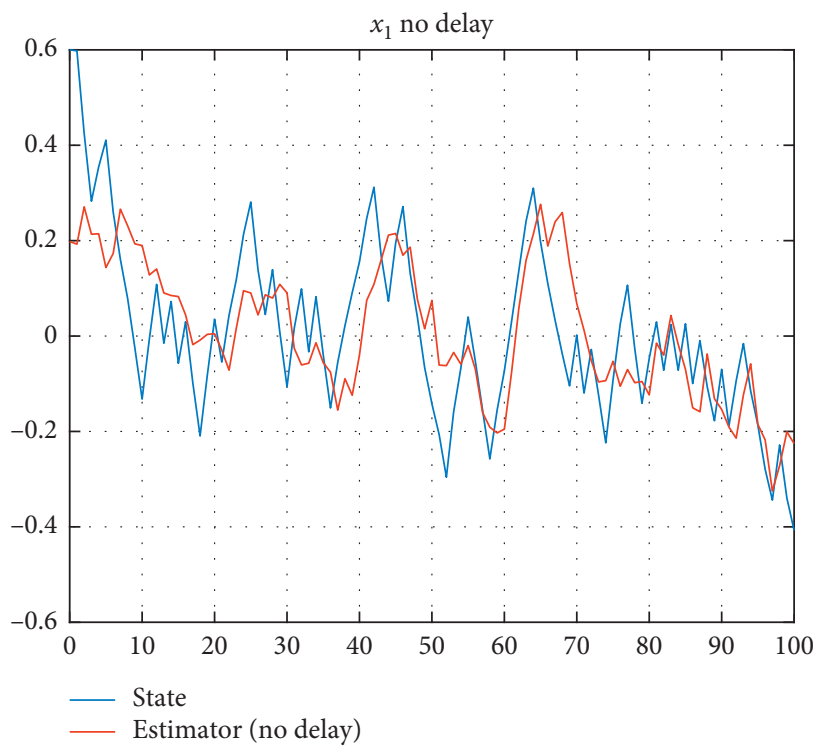

FIgURE 3: The first state component $x_{1}(k)$ and the filter $\hat{x}(k \mid k)$ (no delay).

delays, and Figures 3 and 4 reflect the quadratic estimation results without delays. Then, by comparing Figures 1 and 3 and Figures 2 and 4, it can be observed that the tracking effect of Figures 1 and 2 is better. Therefore, this experiment shows that the information from the measurement delay channel is really important in the design of the quadratic estimator. 


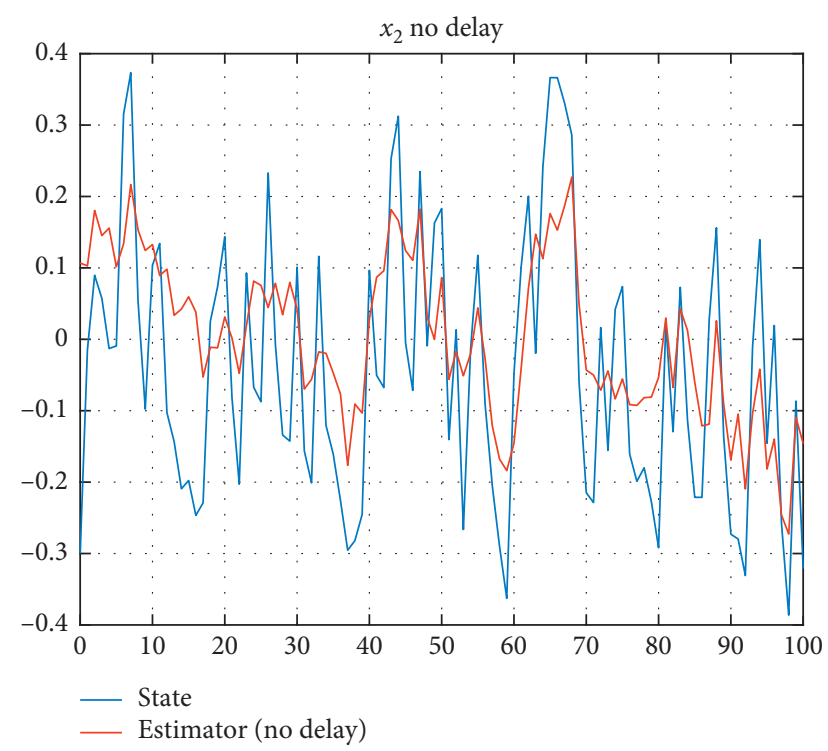

FIgURE 4: The second state component $x_{2}(k)$ and the filter $\widehat{x}(k \mid k)$ (no delay).

\section{Conclusion}

We considered the problem of remote estimation with time delay and multiplicative noise for multichannel systems. At first, we applied the reorganized innovation analysis approach to construct the original delay system into a new delay-free system. Secondly, the delay-free system was reconstructed by the quadratic filtering method to obtain an augmented system. Then, the Kalman filtering theory and the projection formula were used to solve two Riccati equations and one Lyapunov equation for the augmented system, and the quadratic filter for the measurement delay system on the packet loss network was obtained. Finally, simulation experiments proved the effectiveness of the estimator algorithm.

\section{Data Availability}

The simulation program data used to support the proposed approach in numerical example section is available from the corresponding author upon request. No data (except for the simulation program) were used to support this study.

\section{Conflicts of Interest}

The authors express that there are no conflicts of interest concerning with the publication of this work.

\section{Authors' Contributions}

The contribution of all the authors to the writing of this paper is identical. The final manuscript was read and approved by all of them.

\section{Acknowledgments}

This work was supported in part by the National Science Foundation of China (61873152) and the Chinese
Postdoctoral Science Foundation (2019M652424 and 2017M612336).

\section{References}

[1] C. Pang and S. Sun, "Fusion predictors for multisensor stochastic uncertain systems with missing measurements and unknown measurement disturbances," IEEE Sensors Journal, vol. 15, no. 8, pp. 4346-4354, 2015.

[2] X. Song and W. X. Zheng, "Linear estimation for discrete-time periodic systems with unknown measurement input and missing measurements," ISA Transactions, vol. 95, pp. 164172, 2019.

[3] L. Li, D. W. C. Ho, and J. Lu, "Event-based network consensus with communication delays," Nonlinear Dynamics, vol. 87, no. 3, pp. 1847-1858, 2016.

[4] N. E. Nahi, "Optimal recursive estimation with uncertain observation," IEEE Transactions on Information Theory, vol. 15, no. 4, pp. 128-136, 1969.

[5] B. Sinopoli, L. Schenato, M. Franceschetti, K. Poolla, M. I. Jordan, and S. S. Sastry, "Kalman filtering with intermittent observations," IEEE Transactions on Automatic Control, vol. 49, no. 9, pp. 1453-1464, 2004.

[6] H. Zhang, X. Song, and L. Shi, "Convergence and mean square stability of suboptimal estimator for systems with measurement packet dropping," IEEE Transactions on Automatic Control, vol. 57, no. 5, pp. 1248-1253, 2012.

[7] X. Song, Z. Duan, and J. H. Park, "Linear optimal estimation for discrete-time systems with measurement-delay and packet dropping," Applied Mathematics and Computation, vol. 284, pp. 115-124, 2016.

[8] H. Liu, B. Xu, D. Lu, and G. Zhang, "A path planning approach for crowd evacuation in buildings based on improved artificial bee colony algorithm," Applied Soft Computing, vol. 68, pp. 360-376, 2018.

[9] H. Liu, B. Liu, H. Zhang, L. Li, X. Qin, and G. Zhang, "Crowd evacuation simulation approach based on navigation knowledge and two-layer control mechanism," Information Sciences, vol. 436-437, pp. 247-267, 2018.

[10] D. Yang, X. Li, and J. Qiu, "Output tracking control of delayed switched systems via state-dependent switching and dynamic output feedback," Nonlinear Analysis: Hybrid Systems, vol. 32, pp. 294-305, 2019.

[11] A. De Santis, A. Germani, and M. Raimondi, "Optimal quadratic filtering of linear discrete-time non-Gaussian systems," IEEE Transactions on Automatic Control, vol. 40, no. 7, pp. 1274-1278, 1995.

[12] R. Caballero-Águila, A. Hermoso-Carazo, and J. LinaresPérez, "Linear and quadratic estimation using uncertain observations from multiple sensors with correlated uncertainty," Signal Processing, vol. 91, no. 2, pp. 330-337, 2011.

[13] F. Cacace, A. Fasano, and A. Germani, "Quadratic filtering of non-Gaussian systems with intermittent observations," in Proceedings of the 52nd IEEE Conference on Decision and Control, Firenze, Italy, December 2013.

[14] H. Zhao and C. Zhang, "Non-Gaussian noise quadratic estimation for linear discrete-time time-varying systems," Neurocomputing, vol. 174, pp. 921-927, 2016.

[15] F. Cacace, F. Conte, A. Germani, and G. Palombo, "Feedback quadratic filtering," Automatica, vol. 82, pp. 158-164, 2017.

[16] T. H. Lee, H. M. Trinh, and J. H. Park, "Stability analysis of neural networks with time-varying delay by constructing novel Lyapunov functionals," IEEE Transactions on Neural 
Networks and Learning Systems, vol. 29, no. 9, pp. 4238-4247, 2018.

[17] L. B. Wu and J. H. Park, "Adaptive fault-tolerant control of uncertain switched nonaffine nonlinear systems with actuator faults and time delays," IEEE Transactions on Systems, Man, and Cybernetics: Systems, vol. 99, 2019.

[18] X. Li, J. Shen, H. Akca, and R. Rakkiyappan, "LMI-based stability for singularly perturbed nonlinear impulsive differential systems with delays of small parameter," Applied Mathematics and Computation, vol. 250, pp. 798-804, 2015.

[19] B. Niu, D. Wang, M. Liu, X. Song, H. Wang, and P. Duan, "Adaptive neural output-feedback controller design of switched nonlower triangular nonlinear systems with time delays," in Procedings of the IEEE Transactions on Neural Networks and Learning Systems, Piscataway, NJ, USA, December 2020.

[20] Z. Wang, J. Xu, X. Song, and H. Zhang, "Consensus problem in multi-agent systems under delayed information," Neurocomputing, vol. 316, pp. 277-283, 2018.

[21] Z. Wang, H. Zhang, M. Fu, and H. Zhang, "Consensus for high-order multi-agent systems with communication delay," Science China-Information Science, vol. 9, pp. 241-252, 2017.

[22] X. Song and J. H. Park, "Linear optimal estimation for discrete-time measurement delay systems with multichannel multiplicative noise," IEEE Transactions on Circuits and Systems II: Express Briefs, vol. 64, no. 2, pp. 156-160, 2017.

[23] X. Yang, X. Li, X. Li, Q. Xi, and P. Duan, "Review of stability and stabilization for impulsive delayed systems," Mathematical Biosciences \& Engineering, vol. 15, no. 6, pp. 14951515, 2018.

[24] D. Yang, X. Li, J. Shen, and Z. Zhou, "State-dependent switching control of delayed switched systems with stable and unstable modes," Mathematical Methods in the Applied Sciences, vol. 41, no. 16, pp. 6968-6983, 2018.

[25] T. H. Lee and J. H. Park, "New methods of fuzzy sampled-data control for stabilization of chaotic systems," IEEE Transactions on Systems, Man, and Cybernetics: Systems, vol. 48, no. 12, pp. 2026-2034, 2018.

[26] L. B. Wu, J. H. Park, and N. N. Zhao, "Robust adaptive faulttolerant tracking control for nonaffine stochastic nonlinear systems with full state constraints," IEEE Transactions on Cybernetics, vol. 99, 2019.

[27] H. Kwakernaak, "Optimal filtering in linear systems with time delays," IEEE Transactions on Automatic Control, vol. 12, no. 2, pp. 169-173, 1967.

[28] L. Xiao, A. Hassibi, and J. P. How, "Control with random communication delays via a discrete-time jump system approach," in Proceedings of the 2000 American Control Conference, pp. 2199-2204, Chicago, IL, USA, June 2000.

[29] H. Zhang, X. Lu, and D. Cheng, "Optimal estimation for continuous-time systems with delayed measurements," IEEE Transactions on Automatic Control, vol. 51, no. 5, pp. 823-827, 2006. 